\title{
A novel framework for efficient identification of brain cancer region from brain MRI
}

\author{
Parvati Angadi $^{1}$, M. Nagendra ${ }^{2}$, Hanumanthappa M. ${ }^{3}$ \\ ${ }^{1}$ Rayalaseema University, Kurnool, India \\ ${ }^{2}$ Department of Computer Science \& Technology, S K University, India \\ ${ }^{3}$ Department of Computer Science, Bangalore University, India
}

\begin{tabular}{l} 
Article Info \\
\hline Article history: \\
Received Dec 22, 2017 \\
Revised Jul 17, 2018 \\
Accepted Sep 2, 2018 \\
\hline
\end{tabular}

\section{Keywords:}

Accuracy

Brain cancer

Magnetic resonance imaging

Optimization

Tumor detection

\begin{abstract}
Diagnosis of brain cancer using existing imaging techniques, e.g., Magnetic Resonance Imaging (MRI) is shrouded with various degrees of challenges. At present, there are very few significant research models focusing on introducing some novel and unique solutions towards such problems of detection. Moreover, existing techniques are found to have lesser accuracy as compared to other detection schemes. Therefore, the proposed paper presents a framework that introduces a series of simple and computationally costeffective techniques that have assisted in leveraging the accuracy level to a very higher degree. The proposed framework takes the input image and subjects it to non-conventional segmentation mechanism followed by optimizing the performance using directed acyclic graph, Bayesian Network, and neural network. The study outcome of the proposed system shows the significantly higher degree of accuracy in detection performance as compared to frequently existing approaches.
\end{abstract}

Copyright (C) 2019 Institute of Advanced Engineering and Science. All rights reserved.

\section{Corresponding Author:}

Parvati Angadi,

Rayalaseema University,

Kurnool, India.

Email: parvati_angadi@yahoo.com

\section{INTRODUCTION}

Brain cancer is considered as one of the most lethal form of the cancer in human being as where the complications of diagnosis and treatment is exponentially high [1]. Normally, brain cancer is a stage of cancer where a brain region has presence of either lesions or tumor [2]. Lesions are required to be repaired in order to stop being causing further internal hemorrhage. Similarly, brain tumor could be benign or malignant [3]. However, in both the cases, an expensive surgery with complicated surgical equipment, procedures, and highly skilled surgeon is required. All this just begins with the diagnosis stage where normally Magnetic Resonance Imaging (MRI) is highly preferred in initial stage of analysis [4]. However, apart from MRI, there are other imaging techniques too viz. Computed Tomography (CT) scan, Positron Emission Tomography (PET) scan [5]. Although, with the inclusion of technology, radiological studies have significantly improved. However, they have a limitation too [6]. At present, all the imaging techniques could just assists in capturing the region suspected from cancer from very internal-depth (3D Doppler scan with multi-slices), however, there is no such machines or technology who offers conclusive inference of the resultant images. It only depends on the experience of physician that the inference is made considering a slight probability that human error. From research viewpoint, there has been a series of research work being carried out toward cancer detection problem; however majority of the existing research work has actually not focused on brain MRI images. There are very less number of significant research work towards identification of brain tumor in recent times. The most recent survey is carried out 3 year back by Ashwathy [7] to prove that not much progress has happened in this area of cancer detection. The complication in brain cancer is 
very different than other forms of cancer e.g. i) it is highly grayscale image where the tumor regions are very less identified accurately from raw images, ii) performing preprocessing on MRI image could lead to loss of some significant information until and unless it is not assured that it has some noise or errors, iii) normally brain images are highly symmetrical in nature that causes a greater degree of challenges when it comes to feature extraction process, iv) usage of training or machine learning algorithm could result in generation of higher quantity of false positive until and unless a proper stopping function is designed or develop a strategy that can recognize the elite outcomes. Such complications are found very less to be assumed in existing system that causes increasing number of research effort in this direction [8], [9]. It is also seen that segmentation is part and parcel of almost every detection techniques in existing system; however, the techniques are too straightforward. Existing segmentation focuses on just foreground details whereas in order to perform clustering and classification for bigger data set, the background information is equally essential. There is a lot of scope of improvement in addressing the problem of detecting brain tumor which we find very less in number in existing system and thereby it poses a significant research gap.

Therefore, this manuscript offers a very simple optimization technique of brain tumor detection where there is split emphasis on computational efficiency as well as system performance concerning accuracy. The potential features of Neural Network, a Bayesian network, watershed transform-based techniques were harnessed to optimize the accuracy performance. Usage of such techniques results in noniterative and progressive enhancement of the extraction of information that redefines the value of features being extracted that finally results in optimal performance in the identification of brain tumor region extraction from the MRI images of the brain. Section 1.1 discusses the existing literature followed by the discussion of research problems in Section 1.2 and proposed solution in 1.3. Section 2 discusses algorithm implementation followed by the discussion of result analysis in Section 3. Finally, the conclusive remarks are provided in Section 4.

This section discusses about the existing research approach being carried out towards identification of brain cancer. The study carried out by Anitha and Murugavalli [10] have presented a classification technique for investigating brain tumour cells using K-Nearest Neighbor algorithm. Usage of sophisticated image capturing devices was introduced in the study of Baghaei et al. [11] for identification of lesions from the phantom brain images that has exhibited the capability to identify less than $7 \mathrm{~mm}$ size of lesions. Similar direction of the study towards detection process was also carried out by Beddad and Hachemi [12] who have used fuzzy clustering method for classification along with the level set algorithm. Segmentation has been marked as role player in majority of the existing studies. Work done by Demirhan et al. [13] has presented a simple segmentation process using self-organizing map and vector quantization. The technique has also used wavelets for extracting features. Similar problem towards segmentation was also addressed by Hamamci et al. [14] on contrasted image using cellular automata. Haritha [15] have studied various detection methods where k-means algorithm is found to offer better computational performance. Problems pertaining to segmentation and detection was also addressed by Islam et al. [16] using Gabor filters and enhancing AdaBoost algorithm. Kavitha et al. [17] have used feed-forward algorithm and radial basis function to enhance the detection process. Nagtode et al. [18] have used discrete wavelet transform, Gabor transforms, and neural network for classification of cancer. Usage of morphological operators and segmentation for detecting cancer was seen in the work of Nandi [19]. Adoption of time-resolved fluorescence is used by Nie et al. [20] for identification of tumour margin in brain. Perez et al. [21] have studied various techniques of identifying cancer stages of brain from MRI images. Resaeieh et al. [22] have investigated microwave imaging for identification of brain tumor followed by process of performance verification. Salwe et al. [23] have introduced an adaptive process of wavelet implementation where fine windowing operation as well as thresholds was used for detecting tumor cells of brain. Adoption of entropy factor toward similar detection problem was seen in the work carried out by Somwanshi et al. [24] for enhancing the segmentation performance. Dictionary learning was used adopted for incorporating adaptiveness in the detection process followed by usage of sparse reconstruction process as seen in the work carried out by $\mathrm{Su}$ et al. [25]. Subashini and Sahoo [26] used back propagation as well as pulsed_coupled neural network for detection brain tumor cells. Thara and Jasmine [27] used fuzzy K-means clustering as well as C-means clustering in order to perform segmentation that is further followed by neural network for image classification. Usage of morphological operations for addressing similar problem was also seen in the work of Vishnumurthy et al. [28]. Zhang et al. [29] have used microwave imaging and especially Vivaldi antenna for detecting brain tumor. Hence, existing system offers various approaches with benefits as well as limitation. The next section outlines the research problems identified.

The significant research problems are as follows:

a. Existing approaches mainly subjects the input image to either segmentation, clustering, classification, training algorithms without much emphasizing on processing more. 
b. Existing segmentation algorithms only focus on the foreground whereas background information is ignored resulting in cases of false positives.

c. Level of enhancement towards morphological operation is very much limited only to erosion and dilation whereas more potential of the morphological operator could be harnessed.

d. Lesser focus on complex features extraction is another cause of false positive especially when subjected to machine learning algorithms.

Therefore, the problem statement of the proposed study can be stated as "It is highly computationally challenging task to formulate a framework that uses cost-effective techniques to extraction substantial information from the image that could assist in superior identification of brain cancer." The next section discusses the proposed solution.

The proposed study is an extension of our prior contribution [30] where we presently focus on using series of smaller and computationally cost-effective techniques to leverage the accuracy of brain cancer detection.

The methodology involved in designing the first case study is as briefly discussed:

a. The rationale behind Selection of Brain MRI Image: The first part of our study chooses to work on brain MRI images owing to the complication of the type of oncological cancer. Brain cancer is still the most challenging cancerous condition with increased mortality rate, and hence accuracy in diagnosis is highly required. We believe that owing to the complicated form of the brain MRI images; there are possibilities of performing inaccurate feature extraction that may call for false alarm. Therefore, owing to the criticality of the disease condition, we choose to consider brain MRI image as our first case study.

b. Dataset used: MR-TIP database.

c. Process Involved in Design Methodology: The proposed system takes the input of Brain MRI image and applies gradient magnitude for better definition of possible edges Figure 1. This is followed by applying watershed-based transformation scheme for assisting in better segmentation scheme. The processed image is further marked for both foregrounds as well as a background. Finally, a watershed algorithm is applied to perform proper segmentation of the foreground object. A Bayesian Network (BN) is constructed along with Directed Acyclic Graph (DAG) followed by histogram calculation and region merging. This entire process significantly assists in extracting enough information about the normal and abnormal condition of an object. This stage results in the formation of features that are finally subjected to the supervised training carried out by Artificial Neural Network (ANN).

Finally, the detection process is carried out to detect the location of cancer, and the study outcome is assessed using conventional accuracy parameters, e.g., True/false- positive/negative, Sensitivity, Recall rate, Specificity, Precision, F1-Score. The next section discusses the algorithm implementation for developing the proposed detection mechanism.

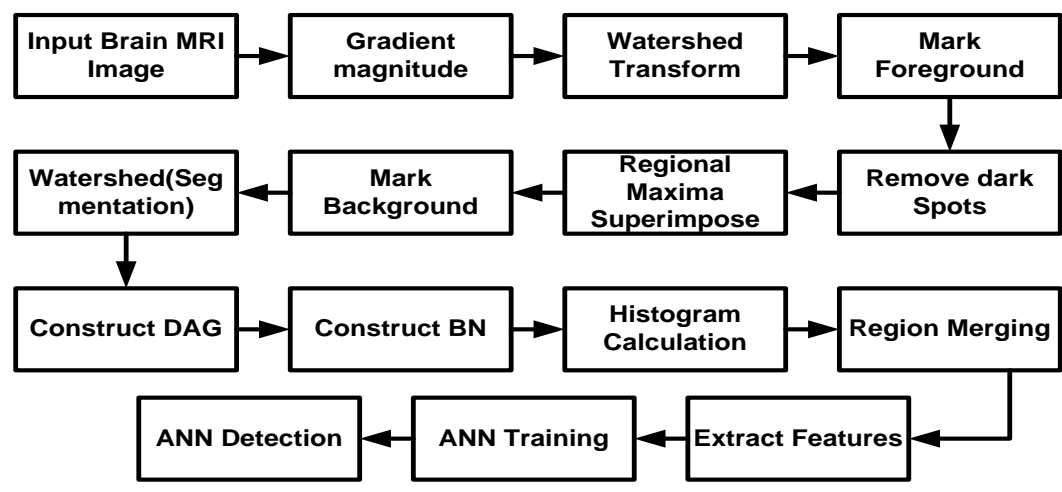

Figure 1. Methodology adopted for 1st case study

\section{ALGORITHM IMPLEMENTATION}

This section discusses the algorithm that performs identification of the cancerous region for a given MRI image of a brain. The algorithm takes the input image I that after processing yields to the final image $\mathrm{I}_{\mathrm{det}}$ with suspected cancerous regions. The significant steps of the algorithm are as follows: 


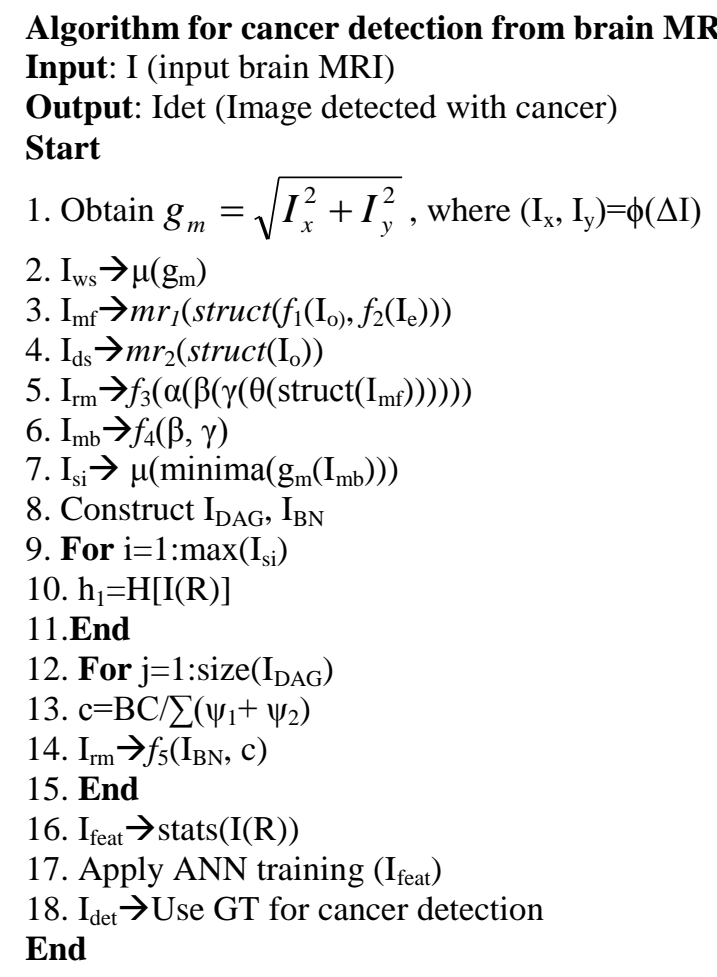

The steps of the above mentioned algorithm is as discussed below: Initially, the input image $I$ undergoes a processing to obtain gradient magnitude $\mathrm{g}_{\mathrm{m}}$ (Line-1). For this purpose, image filter $\phi$ is applied to the image I where a Sobel operator $\Delta$ is applied to extract $I_{x}$ and $I_{y}$. The next part of the algorithm is to apply the watershed transform $\mu$ on the accomplished gradient magnitude $g_{m}$ of input image (Line-2) in order to obtain the transformed image $\mathrm{I}_{\mathrm{ws}}$. The next step of the algorithm is to mark the foreground of an image (Line-3). For this purpose a function $f_{1}$ is used for opening the image morphologically and a function $f_{2}$ is used for eroding the image with respect to the disk morphological structure struct. Finally, a method $m r_{1}$ is used for reconstructing the morphological factors of an image $I$ with respect to $I_{\mathrm{e}}$ in order to obtain finally marked image $I_{\mathrm{mf}}$. This process is also followed by removal of the dark spots by applying morphological closing operator $m r_{2}$ on $I_{0}$ with respect to the structure struct (Line-4). The next part of the algorithm performs dilation operation $\theta$ on foreground marked image $I_{\mathrm{mf}}$ with respect to the structure struct (Line-5). This operation is further succeeded by reconstruction process $\gamma$ carried out morphologically that is further encapsulated by function for computation of complement image $\beta$. Finally, we apply morphological function of region maxima function $\alpha$ and this is further encapsulated by the function $f_{3}$ used for superimposing the foreground in order to obtain superimposed image of regional maxima $\mathrm{I}_{\mathrm{rm}}$. Now the algorithm applies a principle of background marking and for this it obtains the binary image from the prior accomplished steps using the function $\beta$ and $\gamma$ (Line-6).

The obtained image is than explored for its distance between two transformed points in the image that is finally followed by the operation of the watershed transformation operator $f_{4}($ Line- 6$)$ in order to obtain the image where the marked background is obtained $I_{\mathrm{mb}}$. After obtaining both foreground and background image, the next step will be to apply the processing for initiating the segmentation process. For this purpose, initially the image with gradient magnitude $\mathrm{g}_{\mathrm{m}}$ and the concatenation of background image $\mathrm{I}_{\mathrm{mb}}$ and image with regional maxima $I_{\mathrm{rm}}$ and edges and this processing offers second stage gradient magnitude followed by minima-based operation (Line-7). This operation is further subjected to watershed algorithm $\mu$ for better segmentation performance in order to obtain the segmented image $\mathrm{I}_{\mathrm{si}}$. For better computational performance, we apply direct acyclic graph $\mathrm{I}_{\mathrm{DAG}}$ and Bayesian network $\mathrm{I}_{\mathrm{BN}}$ in order to further optimize the detection performance (Line-8)

The mechanism applied for constructing DAG and Bayesian Network is as follows: The first step in construction acyclic graph is to give labels to all the regions and in order to do this a loop is created for all the possible value of labels (extracted from watershed transform) where all the regions are extracted along with morphological dilation operation from where the unique labels are extracted logically. The next step is to apply a probabilistic graphical model, i.e., Bayesian network on obtained directed acyclic graph concerning sizes of the nodes. The next step of the algorithm is to perform region merging operation. In order to do so, a loop is created for all the maximum values of segmented image $I_{s i}$ (Line-9), where regions $R$ are 
checked for their equivalency with the maximum values. A histogram operator $\mathrm{H}$ is then applied to the image with only region information to finally extract the histogram $h_{1}$ (Line-10). This operation is further followed up by computation of merged regions. For this purpose a loop is created for all the maximum values obtained by finding the size of direct acyclic graph, where all the connected components for each segments are found. A histograph of the current node is obtained followed by the computation of the Bhattacharya Coefficient BC (Line-13). Finally, all the connected components $c$ is computed using a simple mathematical expression that uses Bayesian network once again as shown in Line-14. This process results in $\mathrm{I}_{\mathrm{rm}}$ that is a final region merging image. The function $f_{5}$ represents region merging operation and the variables $\psi_{1}$ and $\psi_{2}$ represents cell with $1 \mathrm{x} 1$ dimension and $1 \mathrm{x}$ dimension of all the connected components respectively. This step is followed by extraction of statistical features stats on the regions of an image in order to obtain an image matrix $I_{\text {feat }}$ (Line-16). Finally, neural network is applied on these features (Line-17) where the ground truth GT images were used for assessing the regions that are possibly infected by cancer in resultant image $I_{\text {det }}$ (Line-18). Therefore, the proposed algorithm implements various smaller steps in order to finally perform detection of cancerous portions for a given MRI image of a brain. The next section discusses about the results being obtained

\section{RESULTS ANALYSIS}

This section discusses the result being obtained after implementing the concept written in the prior section. The complete analysis is carried out on images obtained from MR-TIP database [31] where the dimension of the images are approximately $618 \times 630$ with the occupation of $36.2 \mathrm{~KB}$ of memory. The visual outcomes of the proposed system are shown in Figure 2 where it can be seen that input images after subjecting to gradient magnitude loses its contrast and highlights for edge-based information Figure 2(b). Applying watershed algorithm further assists in an effective segmentation process Figure 2(c) and hence it highlights all the possible colors for the transformed image. In the process of segment, it was found that foreground marking Figure 2(d) offers internal changes and not much on superficial changes followed by region merging Figure 2(e). A closer look into the region merging operation in Figure 2(e) shows that the suspected portion of cancer could be located within the region. Therefore, it can be said that true outcome starts coming only after region merging operation. Further implying the direct acyclic graph and Bayesian network, the identification performance of the cancerous regions has tremendously increased as well as it offers better inference of the outcome of the processed image. At the same time, all the missing pixel elements are successfully handled as all the encoding is carried out using Bayesian network. Even from the accuracy viewpoint, the proposed system offers highly enhanced accuracy level as it can mitigate the problem of overfitting to maximize the outcome of identification. Finally, applying neural network training has further optimized the outcome the accuracy level of the proposed system with the shortest iteration of the learning process. The complete optimization was carried out using damped least square algorithm.

For better study effectiveness, we compare the outcome of the proposed study with that of Support Vector Machine (SVM) and K-Nearest Neighbor (KNN) algorithm that is frequently used in existing system for classification purpose. The numerical outcome is shown in Table 1.

Table 1. Comparative Analysis of Existing System

\begin{tabular}{cccc}
\hline Parameters & SVM & KNN & Proposed System \\
\hline Sensitivity/Recall rate & 0.45721 & 0.32872 & 0.653024 \\
Specificity & 0.97695 & 0.93786 & 0.999948 \\
Precision & 0.98675 & 0.97658 & 0.999920 \\
Accuracy & $95 \%$ & $93 \%$ & $99 \%$ \\
\hline
\end{tabular}

The outcome shows that proposed system using neural network offers better accuracy as compared to SVM and KNN. The prime reason behind this is SVM and KNN offers higher computational problem when it comes to performing classification of increased size of data. Moreover, both the algorithm performs optimization till a threshold value whereas in neural network the optimization is more applicable on minimization of errors. This will mean that error minimizing operation is better for neural network in every aspect. Therefore, proposed system will neural network offers enhanced accuracy as compared to existing system. 


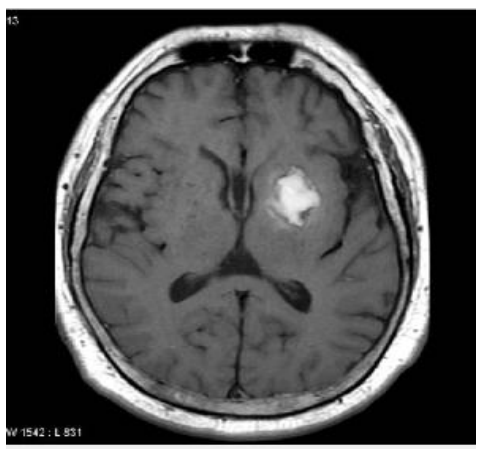

(a)

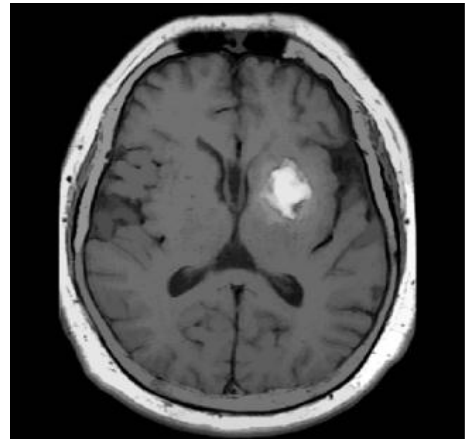

(d)

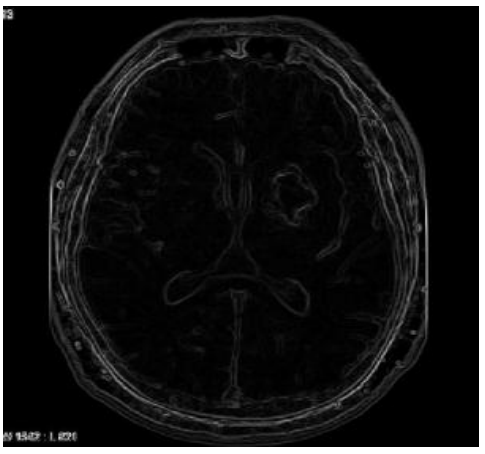

(b)

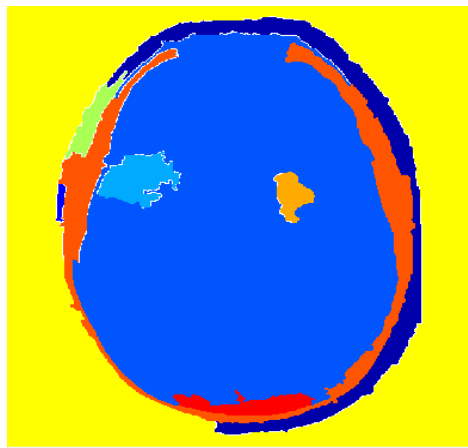

(e)

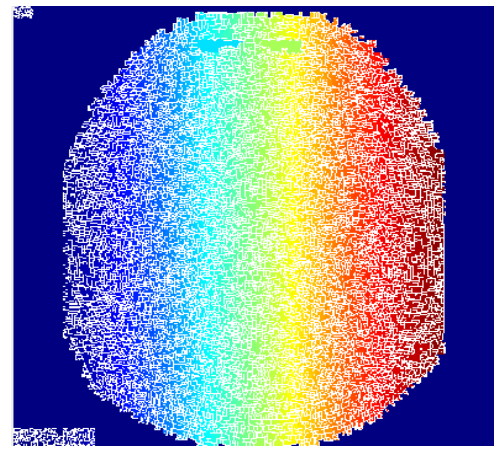

(c)

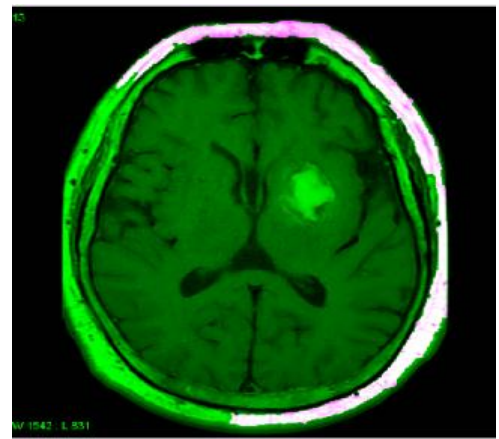

(e)

Figure 2. (a) Input image, (b) Gradient magnitude, (c) Watershed transform, (d) Mark foreground, (e). Region merging, (f) Detection of tumor

\section{CONCLUSION}

This paper has presented a simple and cost-effective technique for enhancing the accuracy level of brain cancer detection using a series of operations. The reason behind its cost effectiveness is because none of the algorithms used in proposed system consumes maximum memory or is dependent on higher number of trained dataset. Most significantly, the response time of proposed system is very less as compared to the existing system. The proposed system also introduces a unique mechanism to perform segmentation where the importance is being shared equally to for marking both foreground and background. Usage of directed acyclic graph as well as Bayesian Network has assisted to improve the extraction of unique information that was used for constructing features. Finally, such features when fed to neural network, we find much-enhanced accuracy performance. The complete analysis is found to offer higher accuracy level in contrast to existing methods. Our future work will be to continue the presence research and include more complications in diagnosis using novel optimization schemes.

\section{REFERENCES}

[1] Adekunle M. Adesina, Tarik Tihan, Christine E. Fuller, Tina Young Poussaint, "Atlas of Pediatric Brain Tumors," Springer, 2016.

[2] Jürg Hodler, Rahel A. Kubik-Huch, Gustav K. von Schulthess, "Diseases of the Brain, Head and Neck, Spine 2016-2019: Diagnostic Imaging," Springer, 2016.

[3] Alessandro Crimi, Bjoern Menze, Oskar Maier, Mauricio Reyes, "Brainlesion: Glioma, Multiple Sclerosis, Stroke and Traumatic Brain Injuries," Springer, 2017.

[4] Christopher M. Collins, "Electromagnetics in Magnetic Resonance Imaging: Physical Principles, Related Applications, and Ongoing Developments," Morgan \& Claypool Publishers, 2016

[5] S. Caorsi, A. Massa, M. Pastorino and A. Rosani, "Microwave Medical Imaging: Potentialities and Limitations of a Stochastic Optimization Technique," in IEEE Transactions on Microwave Theory and Techniques, vol. 52, no. 8, pp. 1909-1916, Aug. 2004.

[6] Rivka R. Colen, Imaging of Brain Tumors, "An Issue of Magnetic Resonance Imaging Clinics of North America," E-Book, Elsevier Health Sciences, 2016. 
[7] S. U. Aswathy, G. G. Deva Dhas and S. S. Kumar, "A Survey on Detection of Brain Tumor from MRI Brain Images," 2014 International Conference on Control, Instrumentation, Communication and Computational Technologies (ICCICCT), Kanyakumari, pp. 871-877, 2014.

[8] Roya Babaie Aghdam, Atieh Sadat Bayat Ghiasi, Parastoo Fatemi, Nazanin Sadat Hashemi, "Challenges in Brain Magnetic Resonance Image Segmentation," American Scientific Research Journal for Engineering, technology, and Sciences, vol.27(1), 2017.

[9] Ivana Despotović, Bart Goossens, and Wilfried Philips, "MRI Segmentation of the Human Brain: Challenges, Methods, and Applications," Computational and Mathematical Methods in Medicine, 2015.

[10] V. Anitha and S. Murugavalli, "Brain Tumour Classification using Two-Tier Classifier with Adaptive Segmentation Technique," in IET Computer Vision, vol. 10(1), pp. 9-17, 2016.

[11] H. Baghaei et al., "Comparison of Brain Phantom Lesion Imaging Capability of the Brain and Whole-Body Modes of the Transformable HOTPET Camera," in IEEE Transactions on Nuclear Science, vol. 58(3), pp. 730-735, Jun 2011.

[12] B. Beddad and K. Hachemi, "Brain Tumor Detection by using a Modified FCM and Level Set algorithms," 2016 4th International Conference on Control Engineering \& Information Technology (CEIT), Hammamet, pp. 1-5, 2016.

[13] A. Demirhan, M. Törü and İ. Güler, "Segmentation of Tumor and Edema Along With Healthy Tissues of Brain Using Wavelets and Neural Networks," in IEEE Journal of Biomedical and Health Informatics, vol. 19(4), pp. 1451-1458, Jul 2015.

[14] A. Hamamci, N. Kucuk, K. Karaman, K. Engin and G. Unal, "Tumor-Cut: Segmentation of Brain Tumors on Contrast Enhanced MR Images for Radiosurgery Applications," in IEEE Transactions on Medical Imaging, vol. 31(3), pp. 790-804, Mar 2012.

[15] D. Haritha, "Comparative Study on Brain Tumor Detection Techniques," 2016 International Conference on Signal Processing, Communication, Power and Embedded System (SCOPES), Paralakhemundi, Odisha, India, pp. 1387-1392, 2016.

[16] A. Islam, S. M. S. Reza and K. M. Iftekharuddin, "Multifractal Texture Estimation for Detection and Segmentation of Brain Tumors," in IEEE Transactions on Biomedical Engineering, vol. 60(11), pp. 3204-3215, Nov 2013.

[17] A. R. Kavitha, C. Chellamuthu and K. Rupa, "An Efficient Approach for Brain Tumour Detection Based on Modified Region Growing and Neural Network in MRI Images," 2012 International Conference on Computing, Electronics and Electrical Technologies (ICCEET), Kumaracoil, pp. 1087-1095, 2012.

[18] S. A. Nagtode, B. B. Potdukhe and P. Morey, "Two Dimensional Discrete Wavelet transform and Probabilistic Neural Network used for Brain Tumor Detection and Classification," 2016 Fifth International Conference on Ecofriendly Computing and Communication Systems (ICECCS), Bhopal, pp. 20-26, 2016.

[19] A. Nandi, "Detection of Human Brain Tumour using MRI Image Segmentation and Morphological Operators," 2015 IEEE International Conference on Computer Graphics, Vision and Information Security (CGVIS), Bhubaneswar, pp. 55-60, 2015.

[20] Z. Nie et al., "Integrated Time-Resolved Fluorescence and Diffuse Reflectance Spectroscopy Instrument for Intraoperative Detection of Brain Tumor Margin," in IEEE Journal of Selected Topics in Quantum Electronics, vol. 22(3), pp. 49-57, May-Jun 2016.

[21] U. Perez, E. Arana, and D. Moratal, "Brain Metastases Detection Algorithms in Magnetic Resonance Imaging," IEEE Latin America Transactions, vol. 14(3), pp.1109-1114, 2016.

[22] S. Ahdi Rezaeieh, A. Zamani and A. M. Abbosh, "3-D Wideband Antenna for Head-Imaging System with Performance Verification in Brain Tumor Detection," in IEEE Antennas and Wireless Propagation Letters, vol. 14, no. 3, pp. 910-914, 2015.

[23] S. Salwe, R. Raut and P. Hajare, "Brain Tumor Pixels Detection using Adaptive Wavelet Based Histogram Thresholding and Fine Windowing," 2016 International Conference on Information Technology (InCITe) - The Next Generation IT Summit on the Theme - Internet of Things: Connect your Worlds, Noida, pp. 256-260, 2016.

[24] D. Somwanshi, A. Kumar, P. Sharma and D. Joshi, "An Efficient Brain Tumor Detection from MRI Images using Entropy Measures," 2016 International Conference on Recent Advances and Innovations in Engineering (ICRAIE), Jaipur, pp. 1-5, 2016.

[25] H. Su, F. Xing and L. Yang, "Robust Cell Detection of Histopathological Brain Tumor Images Using Sparse Reconstruction and Adaptive Dictionary Selection," in IEEE Transactions on Medical Imaging, vol. 35(6), pp. 1575-1586, Jun 2016.

[26] M. M. Subashini and S. K. Sahoo, "Brain Tumour Detection using Pulse Coupled Neural Network (PCNN) and Back Propagation Network," IET Chennai 3rd International on Sustainable Energy and Intelligent Systems (SEISCON 2012), Tiruchengode, pp. 1-6, 2012.

[27] K. S. Thara and K. Jasmine, "Brain Tumour Detection in MRI Images using PNN and GRNN," 2016 International Conference on Wireless Communications, Signal Processing and Networking (WiSPNET), Chennai, pp. 1504-1510, 2016.

[28] T. D. Vishnumurthy, H. S. Mohana and V. A. Meshram, "Automatic Segmentation of Brain MRI Images and Tumor Detection using Morphological Techniques," 2016 International Conference on Electrical, Electronics, Communication, Computer and Optimization Techniques (ICEECCOT), Mysuru, India, pp. 6-11, 2016.

[29] H. Zhang, B. Flynn, A. T. Erdogan and T. Arslan, "Microwave Imaging for Brain Tumour Detection using an UWB Vivaldi Antenna array," 2012 Loughborough Antennas \& Propagation Conference (LAPC), Loughborough, pp. 1-4, 2012. 
[30] P. N. Angadi and P. K. Srimani, "DODOR: Dual Optimization for Detection of Oncological Region in Radiological Image," 2015 International Conference on Emerging Research in Electronics, Computer Science and Technology (ICERECT), Mandya, pp. 198-203, 2015.

[31] "Brain MRI", http://www.mr-tip.com/serv1.php?type=db1\&dbs=Brain\%20MRI, Retrieved on 15 ${ }^{\text {th }}$ July, 2017.

\section{BIOGRAPHIES OF AUTHORS}

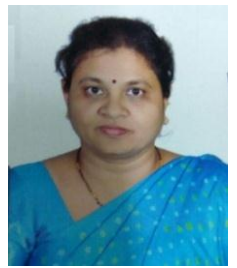

Parvati Angadi, is a research scholar of Rayalaseema University having 20 years of Industry and Teaching Experience. She has published over 20 papers her area of interest focuses mainly on Automation.

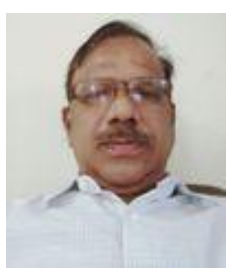

Dr. M Nagendra, is professor and HOD at Department of Computer Science and Technology, S.K. University. He has a rich academic experience of over 28 years. His areas of interest are Computer Networks and Software Engineering. He is an author of over 20 publications and he mentored $6 \mathrm{Ph} . \mathrm{D}$ students. He has been teaching variety of papers in Computer Science for MCA and M.Sc (CS) courses at Dept of Computer Science and Technology, S.K. University, Anantpuramu

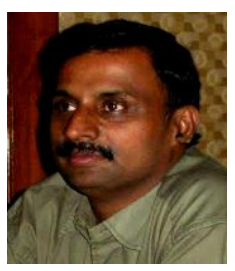

Dr. Hanumanthappa M, is professor at Department of Computer Science, Bangalore University. Having 18 years of teaching, administration and industry experience. His research focuses on Information retrieval, Data mining, Network security and Natural language processing. $\mathrm{He}$ is author of 5 books and over 95 publications. He has adjudicated nearly 50 Ph.D and M.Phil thesis. He has many distinctions conferred. 